\title{
新型苯并咪唑环蕃的合成与晶体结构研究
}

\author{
侯晓萌徐林飞唐雪松宋阳白银娟*
}

(合成与天然功能分子化学教育部重点实验室 西北大学化学与材料科学学院 西安 710069)

\begin{abstract}
摘要 以二溴甲基苯 $(o, m, p)$ 为原料, 分别与 2-巯基苯并咪唑环化合成相应的含硫苯并咪唑环蕃, 实验发现二溴甲基取 代位置对反应结果有很大影响, $o$-位取代很易生成[1:1]型环化产物, $p$-位取代则主要得到链状产物, $m$-位取代环化产物 较复杂，但以[2:2]型环化产物为主. 产物结构通过 ${ }^{1} \mathrm{H}$ NMR, IR, HRMS 进行了表征, 并得到了 1-3a 的 X 射线单晶衍射 晶体结构, 对其晶体结构进行了分析.

关键词 苯并咪唑环蕃; 合成; 表征; 晶体结构
\end{abstract}

\section{Synthesis and Crystal Structure of Novel Benzimidazole Cyclophanes}

\author{
Hou, Xiaomeng Xu, Linfei Tang, Xuesong Song, Yang Bai, Yinjuan* \\ (Key Laboratory of Synthetic and Natural Functional Molecule Chemistry of Ministry of Education, \\ College of Chemistry \& Materials Science, Northwest University, Xi'an 710069)
}

\begin{abstract}
The novel sulfur-containing benzimidazole cyclophanes were synthesized from bis(bromomethyl)benzene $(o, m, p)$ and 2-benzimidazolinthion, respectively. The reaction showed that bromomethyl positions effected the aim compounds very much. It was easy for $o$-substituted to generate the [1:1] type cyclization product. $p$-Substituted obtained chain product, and $m$-substituted benzene pruduced the [2:2] type cyclization compound as main product. The lead compounds were characterized by ${ }^{1} \mathrm{H}$ NMR, IR, HRMS, and the structure of 1-3a was determined by X-ray diffraction method.
\end{abstract}

Keywords benzimidazole cyclophane; synthesis; characterization; crystal structure

环蕃是超分子化学的一个重要分支, 随着这一领域 的深入研究，该化合物不仅表现出环糊精、冠(穴)醚、 杯芳烃和多齿配体的一些特点, 而且在合成方法及结构 修饰上更为多样化, 能更好发挥氢键、疏水作用、静电 作用、 $\pi-\pi$ 、阳离子 $-\pi$ 的协同效应 ${ }^{[1 \sim 4]}$. 苯并咪唑环蕃是 环蕃化学领域中新型的模拟酶模型, 一方面环蕃的环形 空腔具有疏水性, 另一方面苯并咪唑环上的氮原子具有 良好的反应活性, 可以通过氮原子的质子化、季胺化等 引入活性基团 ${ }^{[5]}$, 从而改善该类化合物的性质, 形成不 同包结性能的主体, 俘获不同种类的客体分子, 与客体 分子形成超分子体系, 实现酶功能模拟与分子识别 ${ }^{[6]}$. 另外, 由于杂原子 $(\mathrm{S}, \mathrm{N}, \mathrm{O})$ 的引入而形成的杂原子桥联 环蕃, 使得环蕃的主体结构和键合性能发生改变, 可以 识别不同的客体分子 ${ }^{[5,7]}$.
苯并咪唑环蕃发展至今, 其结构日益复杂精巧, 从 包含两个苯并咪唑鎓离子的小环蕃 ${ }^{[8,9]}$ 到包含三个苯并 咪唑鎓离子的三维洞状环蕃 ${ }^{[10]}$, 再到包含四个苯并咪 唑鎓离子的大环蕃 ${ }^{[11]}$ 等都已见报道. 在结构设计合成 过程中, 通过改变连接链的结构 ${ }^{[12,13]}$ 而赋予了环蕃不同 的性能使其用途也越来越广. 其常用的合成路线有三 种: (1)一步成环法: 双功能基烷基试剂和苯并咪唑类化 合物直接环化得到苯并咪唑环蕃; (2)分步成环法: 先由 双功能基烷基试剂和苯并咪唑类化合物反应形成双苯 并咪唑化合物, 而后苯并咪唑的另一个氮原子再与另一 双功能基烷基试剂直接季胺化成环, 得到苯并咪唑环 蕃; (3)在合成苯并咪唑环的同时合成苯并咪唑环蕃 ${ }^{[14]}$. 在反应上常通过缩合反应脱去卤化氢 ${ }^{[15 \sim 17]}$ 、对甲苯磺 酸 ${ }^{[18]}$ 和三氟甲磺酸 ${ }^{[19]}$ 等小分子完成链的链接或关环.

\footnotetext{
* E-mail: baiyinjuan@sina.com

Received September 28, 2012; revised November 29, 2012; published online December 10, 2012.

Project supported by the National Natural Science Foundation of China (No. 21042002) and the Natural Science Foundation of Shaanxi Province (No. 2010JM2001).

国家自然科学基金(No. 21042002)和陕西省自然科学基金(No. 2010JM2001)资助项目.
} 
反应除常采用的传统溶液法外，微波无溶剂促进法 ${ }^{[20]}$ 等方法也见诸报道. 本文对新型含硫苯并咪唑环蕃的合 成及其晶体结构进行了研究.

\section{1 实验部分}

\section{1 试剂及仪器}

北京光电设备厂 X-4 显微熔点仪, 温度计未校正; Varian INOVA-400 MHz 超导核磁共振仪, TMS 为内标; Brucker EQUIOX-55 红外光谱仪, $\mathrm{KBr}$ 压片; Brucker micrOTOF-QII 质谱仪; Bruker Smart-APEXII CCD 单晶 $X$ 射线衍射仪; 所用试剂均为分析纯.

\section{2 化合物的合成}

\subsection{1 化合物 1-1 的合成}

在 $100 \mathrm{~mL}$ 三颈瓶中加入 $28 \mathrm{~mL}$ 水, $10.81 \mathrm{~g}(0.1 \mathrm{~mol})$ 邻苯二胺, $0.17 \mathrm{~g}$ 十二烷基三甲基溴化铵与 $24.5 \mathrm{~mL}$ 碳酸 钠溶液 (30\%), 摚拌均匀. 滴加 $7.0 \mathrm{~mL}(0.12 \mathrm{~mol})$ 二硫化 碳, $0.5 \mathrm{~h}$ 滴完, 控制温度在 $33 \sim 35{ }^{\circ} \mathrm{C}$, 反应 $2 \mathrm{~h}$; 之后在 $2 \mathrm{~h}$ 内逐渐升温至 $95 \sim 98{ }^{\circ} \mathrm{C}$, 至无硫化氢放出. 用醋酸 调整 $\mathrm{pH}$ 呈中性, 抽滤, 水洗, 干燥, 用水进一步重结晶, 得 $13.40 \mathrm{~g}$ 白色固体 1-1, 产率 89.3\%. m.p. 301 305 ${ }^{\circ} \mathrm{C}$ (文献值 ${ }^{[21]} 301 \sim 303{ }^{\circ} \mathrm{C}$ ).

\subsection{2 化合物 1-2 的合成}

在 $250 \mathrm{~mL}$ 圆底烧瓶中加入 $7.5 \mathrm{~mL}(0.06 \mathrm{~mol})$ 邻二 甲苯, $21.36 \mathrm{~g}(0.12 \mathrm{~mol}) \mathrm{NBS}, 0.40 \mathrm{~g}$ 过氧化苯甲酰以及 $60 \mathrm{~mL} \mathrm{CCl}_{4}$, 回流 $8 \mathrm{~h}$. 冷却, 过滤, 浓缩滤液结晶, 抽 滤, 用正已烷重结晶, 干燥得到 $12.10 \mathrm{~g}$ 白色固体 1-2a, 产率 $77.1 \%$, m.p. $92 \sim 94{ }^{\circ} \mathrm{C}$ (文献值 ${ }^{[22]} 88 \sim 90{ }^{\circ} \mathrm{C}$ ).

同法合成化合物 1-2b 和 1-2c: 得 $9.50 \mathrm{~g}$ 白色固体 1-2b, 产率 $60.5 \%$, m.p. $74 \sim 76{ }^{\circ} \mathrm{C}$ (文献值 ${ }^{[23]} 76{ }^{\circ} \mathrm{C}$ ); 得 $8.70 \mathrm{~g}$ 黄色固体 1-2c, 产率 $55.4 \%$, m.p. $142 \sim 144{ }^{\circ} \mathrm{C}$ (文 献值 ${ }^{[24} 142 \sim 143{ }^{\circ} \mathrm{C}$ ).

\subsection{3 化合物 1-3 的合成}

在 $500 \mathrm{~mL}$ 三颈瓶中加入 $0.96 \mathrm{~g}(0.0064 \mathrm{~mol}) 2$-颈基 苯并咪唑、 $0.48 \mathrm{~g}(0.02 \mathrm{~mol}) \mathrm{NaH}$ 和 $300 \mathrm{~mL}$ THF, 加热 回流 $1 \mathrm{~h}$ 后冷却至室温. 在 $100 \mathrm{~mL}$ THF 中溶解 $1.70 \mathrm{~g}$ (0.0064 mol)邻二溴甲基苯, 常温下用滴液漏斗在 $0.5 \mathrm{~h}$ 内滴加到烧瓶中, 加热回流 $24 \mathrm{~h}$ 冷却至室温, 加入冰水 除去残余的 $\mathrm{NaH}$, 蒸馏回收 THF. 残留固体用 $50 \mathrm{~mL}$ 氯 仿溶解, 抽滤, 滤液用 $25 \mathrm{~mL}$ 饱和食盐水洗涤, 有机层 用无水硫酸镁干燥, 减压蒸馏除去氯仿, 剩余固体用层 析柱进行分离 $\left(V_{\text {石湖酸 }}: V_{\text {丙雨 }}=2: 1\right)$, 得 $0.52 \mathrm{~g}$ 黄色固体 1-3a, 产率 32.5\%. m.p. $157 \sim 159{ }^{\circ} \mathrm{C} ;{ }^{1} \mathrm{H}$ NMR $\left(\mathrm{CDCl}_{3}\right.$, $400 \mathrm{MHz}) \delta$ : 4.39 (s, 2H), 5.35 (s, 2H), $7.31 \sim 7.18(\mathrm{~m}$, $3 \mathrm{H}), 7.43 \sim 7.35(\mathrm{~m}, 4 \mathrm{H}), 7.88(\mathrm{~d}, J=7.21 \mathrm{~Hz}, \mathrm{H})$; IR
(KBr) $v$ : 3055, 2939, 1600, 1417, 1241, $741 \mathrm{~cm}^{-1}$; HRMS calcd for $\mathrm{C}_{15} \mathrm{H}_{12} \mathrm{~N}_{2} \mathrm{~S}[\mathrm{M}+\mathrm{H}]^{+}$253.0794, found 253.0790.

同法合成 1-3 b, 剩余固体用层析柱进行分离 $\left(V_{\text {氯仿 }}: V_{\text {甲睟 }}=30: 1\right)$, 得到 $0.37 \mathrm{~g}$ 淡黄色的固体 1-3b. ${ }^{1} \mathrm{H}$ NMR $\left(\mathrm{CDCl}_{3}, 400 \mathrm{MHz}\right) \delta: 4.51$ (s, 2H), $4.95(\mathrm{~s}, 2 \mathrm{H})$, $7.42 \sim 7.27(\mathrm{~m}, 4 \mathrm{H}), 7.56(\mathrm{~s}, 1 \mathrm{H}), 7.67 \sim 7.64(\mathrm{~m}, 3 \mathrm{H})$; IR (KBr) v: 2993, 2919, 2802, 1622, 1515, 1363, 742, 712 $\mathrm{cm}^{-1}$; HRMS calcd for $\mathrm{C}_{15} \mathrm{H}_{12} \mathrm{~N}_{2} \mathrm{~S}[\mathrm{M}+\mathrm{H}]^{+}[1: 1]$ 型 253.0794, found 253.0797; calcd for $\mathrm{C}_{30} \mathrm{H}_{24} \mathrm{~N}_{4} \mathrm{~S}_{2}[\mathrm{M}+\mathrm{H}]^{+}$ [2:2]型 505.1515, found 505.1536; calcd for $\mathrm{C}_{45} \mathrm{H}_{36} \mathrm{~N}_{6} \mathrm{~S}_{3}$ $[\mathrm{M}+\mathrm{H}]^{+}$[3:3]型 757.2236, found 757.2224; calcd for $\mathrm{C}_{60} \mathrm{H}_{48} \mathrm{~N}_{8} \mathrm{~S}_{4} \quad[\mathrm{M}+\mathrm{H}]{ }^{+}$[4:4] 型 1009.2958, found 1009.2986.

\subsection{4 化合物 2-1 的合成}

在 $250 \mathrm{~mL}$ 圆底烧瓶中加入 $3.76 \mathrm{~g}(0.025 \mathrm{~mol}) 2$-颈 基苯并咪唑, $3.33 \mathrm{~g}(0.0126 \mathrm{~mol})$ 间二溴甲苯和 $1.57 \mathrm{~g}$ $\mathrm{KOH}(0.028 \mathrm{~mol})$, 以及 $50 \mathrm{~mL} \mathrm{DMF}$ 和 $20 \mathrm{~mL} \mathrm{H}_{2} \mathrm{O}$, 室温 搅拌 $6 \mathrm{~h}$, 再加入 $70 \mathrm{~mL} \mathrm{H}_{2} \mathrm{O}$, 继续摚拌 $1 \mathrm{~h}$, 过滤, 用乙 酸乙酯-正己烷 $(V: V=1: 1)$ 重结晶, 得 $2.70 \mathrm{~g}$ 白色固 体 2-1, 产率 54\%. m.p. $265 \sim 267{ }^{\circ} \mathrm{C} ;{ }^{1} \mathrm{H}$ NMR (DMSO- $\left.d_{6}, 400 \mathrm{MHz}\right) \delta: 4.74(\mathrm{~s}, 4 \mathrm{H}), 7.46 \sim 7.23(\mathrm{~m}, 8 \mathrm{H})$, $7.56(\mathrm{~s}, 1 \mathrm{H}), 7.67 \sim 7.65$ (m, 3H); IR (KBr) v: 2991, 2899, 2830, 1620, 1512, 1189, 738, $710 \mathrm{~cm}^{-1}$; HRMS calcd for $\mathrm{C}_{22} \mathrm{H}_{18} \mathrm{~N}_{4} \mathrm{~S}_{2}[\mathrm{M}+\mathrm{H}]^{+}$403.1046, found 403.1028.

\subsection{5 化合物 2-2 的合成}

在 $500 \mathrm{~mL}$ 圆底烧瓶中加入 $0.81 \mathrm{~g}(0.002 \mathrm{~mol})$ 化合 物 2-4b, $0.66 \mathrm{~g}(0.0025 \mathrm{~mol})$ 间二溴甲苯和 $200 \mathrm{~mL}$ 乙腈, 回流 $12 \mathrm{~h}$, 冷却后过滤, 得 $0.45 \mathrm{~g}$ 白色固体 2-2, 产率 $45 \%$. m.p. $>300{ }^{\circ} \mathrm{C} ;{ }^{1} \mathrm{H}$ NMR (DMSO- $\left.d_{6}, 400 \mathrm{MHz}\right) \delta$ : $4.53 \sim 4.45(\mathrm{~m}, 4 \mathrm{H}), 5.08 \sim 4.93(\mathrm{~m}, 4 \mathrm{H}), 7.70 \sim 6.71(\mathrm{~m}$, $16 \mathrm{H})$; IR (KBr) v: 2954, 2924, 2853, 1599, 1461, 1364, $1154,775,701 \mathrm{~cm}^{-1}$; HRMS calcd for $\mathrm{C}_{30} \mathrm{H}_{24} \mathrm{~N}_{4} \mathrm{~S}_{2}[\mathrm{M}+$ $\mathrm{H}]^{+}$505.1515, found 505.1536.

\section{3 化合物 1-3a 的晶体培养和结构测试}

在 $25 \mathrm{~mL}$ 的称量瓶中, 将化合物 1-3a 溶解在氯仿中 配成适当的稀溶液, 用保鲜膜覆盖瓶口, 室温静置待溶 剂自然挥发得所需晶体. 选取合适的单晶样品置于 Bruker Smart-APEXII CCD 单晶 X 射线衍射仪上, 用经 石墨单色器单色纯化的 Mo Ka 射线 $(\lambda=0.071073 \mathrm{~nm})$ 为 光源, 以 $\omega$ 扫描方式扫描, 收集衍射数据, 全部强度数 据经 Lp 校正和吸收校正. 结构由直接法通过 SHELXTL 软件解出. 全部非氢原子坐标、各向异性温度因子和氢 原子坐标及各向同性温度因子经全矩阵最小二乘法 $F_{2}$ 精修修正至收玫. 所有相关计算均使用 SHELXL-97 程 序包完成. 全部非氢原子经 Fourier 合成及差值电子密 
度函数修正, 全部氢原子坐标从差值电子密度函数并结 合几何分析方法获得.

\section{2 结果与讨论}

\section{1 合成}

Scheme 1 所示是化合物 1-3 的合成路线, 以邻苯二 胺和二硫化碳为原料合成 2-颈基苯并咪唑 1-1 ${ }^{[25]}$, 再以 二甲苯和 NBS 原料合成相应的二溴甲基苯 1-2, 然后在 $\mathrm{NaH}$ 作用下环化得到苯并咪唑环蕃目标化合物 $\mathbf{1 - 3}^{[11]}$. 实验发现二溴甲基取代位置对反应结果有很大影响, $o-$ 位取代很易发生反应生成[1:1]型环化产物, 该产物经柱 层析纯化效果很好, 并容易结晶, 经培养得到了单晶. $p$-位取代则主要得到链状混合物, 这与苯环取代基处在 对位，并只有一个亚甲基有关. $m$-位取代环化产物较复 杂, 反应粗产物经 MALDI-TOF MS 表明生成了 [1:1] $(M=253.0797)$ 型、[2:2]型 $(M=505.1536) 、[3: 3]$ 型 $(M=$ 757.2224)以及[4:4]型 $(M=1009.2986)$ 环化产物, 分离困 难, 但以[2:2]型为主.

为了控制环化类型, 我们采用了分步合成法, 通过 改变反应物配比、反应浓度和反应条件, 使 2-颈基苯并 咪唑和间二溴甲基苯在 $\mathrm{KOH}$ 作用下反应生成了含两个 苯并咪唑基的中间产物, 再和另一分子间二溴甲基苯反 应，在乙腈中回流关环生成了 [2:2]型苯并咪唑环蕃 $\mathbf{2 - 2}$ (Scheme 2).

2-颈基苯并咪唑存在硫酮式和硫醇式结构互变异 构体, 可通过质子迁移而发生结构互变 ${ }^{[26]}$ (Eq. 1). 文献
[27～30]报道的 2-颈基苯并咪唑的取代反应大多数都先 取代颈基上的氢，而苯并咪唑上 $\mathrm{N}$ 原子有良好的反应活 性, 通过这两个杂原子的作用进行修饰, 可以改善环蕃 的性质，形成具有不同包结性能的主体，俘获不同种类 的客体分子，与客体形成超分子体系，实现酶功能模拟 和分子识别.

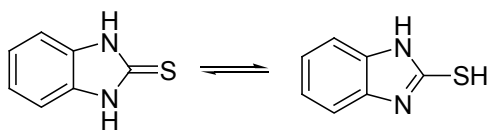

\section{2 化合物波谱分析}

从化合物 1-3a 的 ${ }^{1} \mathrm{H}$ NMR 可以看出 $\delta 7.31 \sim 7.18$ $(\mathrm{m}, 3 \mathrm{H}), 7.43 \sim 7.35(\mathrm{~m}, 4 \mathrm{H}), 7.88(\mathrm{~d}, J=7.21 \mathrm{~Hz}, \mathrm{H})$ 为苯 环上的 8 个氢, $\delta 5.35(\mathrm{~s}, 2 \mathrm{H})$ 为与 $\mathrm{N}$ 原子相邻的亚甲基上 的两个氢, $\delta 4.39(\mathrm{~s}, 2 \mathrm{H})$ 为与 $\mathrm{S}$ 原子相邻的亚甲基上的两 个氢, 由于 $\mathrm{N}$ 原子和 $\mathrm{S}$ 原子的吸电子诱导效应使得亚甲 基上质子周围的电子云密度减弱，屏蔽作用减弱，质子 共振吸收移向低场，化学位移值增大，而且 $\mathrm{N}$ 原子的吸 电子效应强于 $\mathrm{S}$ 原子，所以与 $\mathrm{N}$ 原子相邻的亚甲基上的 两个氢的化学位移增大更多. 由红外图谱可以看出, 在 $3055 \mathrm{~cm}^{-1}$ 处为苯环上质子的伸缩振动吸收峰, 1600 $\mathrm{cm}^{-1}$ 处为芳环骨架的振动吸收峰, $741 \mathrm{~cm}^{-1}$ 处说明苯环 是邻二取代. 飞行质谱测得分子量为 253.0790 , 与单晶 结构一致.

化合物 1-3b 虽然为混合物，但由于结构单元相同， 从 ${ }^{1} \mathrm{H}$ NMR 难以分辨各个化合物. 按照最小比例可见 $\delta$

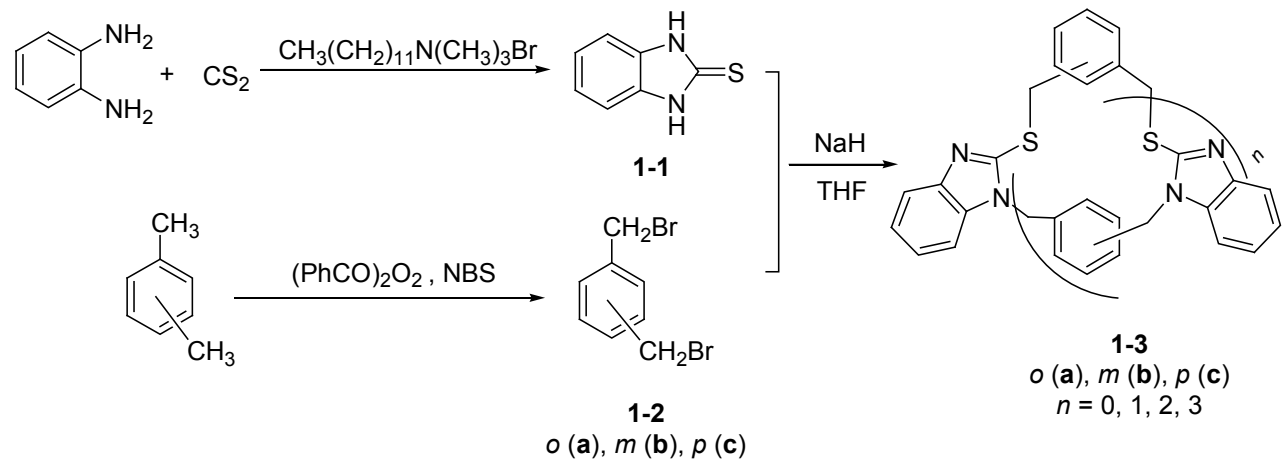

Scheme 1

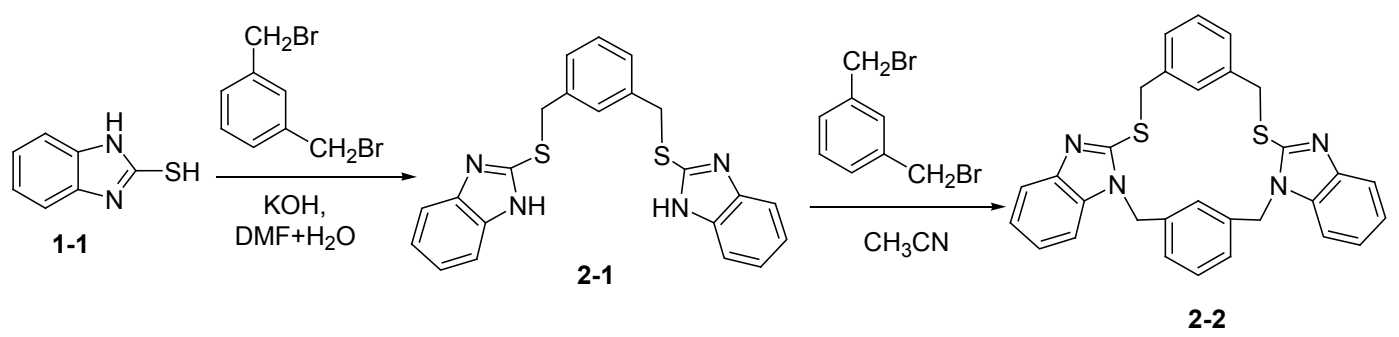

Scheme 2 
$7.42 \sim 7.27(\mathrm{~m}, 4 \mathrm{H}), 7.56(\mathrm{~s}, 1 \mathrm{H}), 7.67 \sim 7.64(\mathrm{~m}, 3 \mathrm{H})$ 为苯 环上的 8 个氢, $\delta 4.95(\mathrm{~s}, 2 \mathrm{H})$ 为与 $\mathrm{N}$ 原子相邻的亚甲基上 的两个氢, $\delta 4.51(\mathrm{~s}, 2 \mathrm{H})$ 为与 $\mathrm{S}$ 原子相邻的亚甲基上的两 个氢; 由红外图谱可以看出, $1622 \mathrm{~cm}^{-1}$ 处为咪唑环中 $\mathrm{C}=\mathrm{N}$ 的伸缩振动吸收峰, $712 \mathrm{~cm}^{-1}$ 处说明苯环是间二 取代, 产物以[2:2]型环化产物为主. 为此采用分步合成 法制备了间位取代环蕃 2-2 (即 1-3b, $n=1$ ), 并通过 ${ }^{1} \mathrm{H}$ NMR, IR, HRMS 得以证实.

\section{3 晶体结构}

据文献报道, 随着环蕃连接链的长度的增加, 分子 面越趋于平面化, 不利于环蕃对底物分子的包结, 桥联 芳环的链越短, 越易形成较好的分子空腔, 使环蕃具有 特定的分子识别性能 ${ }^{[31]}$. 化合物 1-3a 的苯环和苯并咪 唑环通过一条由杂原子 $\mathrm{S}$ 和一个亚甲基组成链以及另一 个亚甲基连接成环, 从晶体结构可以看出, 该化合物的 苯环和苯并咪唑环分别处于两个不同的平面内, 结构呈 明显的 $\mathrm{V}$ 字型, 两个面的夹角为 $113.2^{\circ}$. 因此, 该化合 物具有一定深度的空腔, 并且环上的杂原子 $\mathrm{S}, \mathrm{N}$ 通过与 底物分子的接触可以对底物分子的多重识别和多部分 包结 ${ }^{[32]}$.

化合物 1-3a 的晶体分子式为 $\mathrm{C}_{46} \mathrm{H}_{37} \mathrm{Cl}_{3} \mathrm{~N}_{6} \mathrm{~S}_{3}$, 分子 量为 876.35. 晶胞中有三个该化合物分子和一个溶剂氯 仿分子. 单晶 $\mathrm{X}$ 射线衍射测试表明该晶体属单斜晶系, $P 21 / c$ 空间群. 化合物晶体结构见图 1, 晶胞堆积图见图 2 , 部分键长键角数据分别列于表 $1 . S(1)-C(1)$ 和 $\mathrm{S}(1)-\mathrm{C}(15)$ 的键长分别为 $0.1732(6)$ 和 $0.1824(6) \mathrm{nm}$, 以 上键长的差别是因为 $\mathrm{C}(1)$ 是苯并咪唑 2 位的碳原子, 与 两个 $\mathrm{N}$ 原子直接相连, 而 $\mathrm{C}(15)$ 是桥连亚甲基的碳原子, 其与苯环直接相连, 由于 $\mathrm{N}$ 原子电负性的影响, 使得 $\mathrm{S}(1)-\mathrm{C}(1)$ 的键长比较短. $\mathrm{N}(1)-\mathrm{C}(8)-\mathrm{C}(9)$ 和 $\mathrm{C}(1)-$ $\mathrm{S}(1)-\mathrm{C}(15)$ 的键角分别为 $112.6(4)^{\circ}$ 和 104.9(3) ${ }^{\circ}$. 在化合
物的晶体结构中，处于中间位置的苯并咪唑环分别和相 邻的一个分子中的 $\mathrm{C}(10)-\mathrm{H}(10)$ 以及另一个相邻分子 中的 $\mathrm{C}(15)-\mathrm{H}(15)$ 存在 $\mathrm{C} \cdots \mathrm{H} \cdots \pi$ 相互作用. 同时, 处于 中间位置的分子的苯并咪唑环和相邻的一个分子的苯 并咪唑环存在 $\pi \cdots \pi$ 堆积作用, 而且其苯环也和另一个 相邻分子的苯环存在 $\pi \cdots \pi$ 堆积作用. 该晶体在剑桥晶 体中心分配的 CCDC 号码为 908143 .

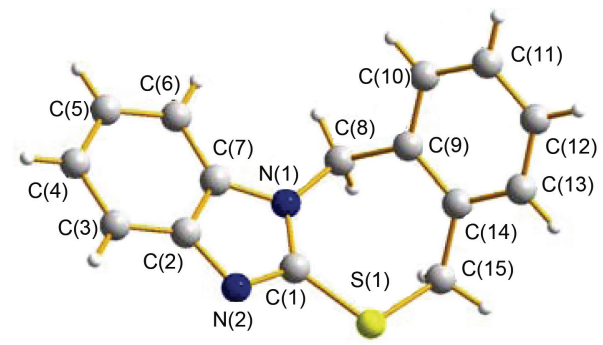

图 1 化合物 1-3a 的晶体结构图 Figure 1 Crystal structure of 1-3a

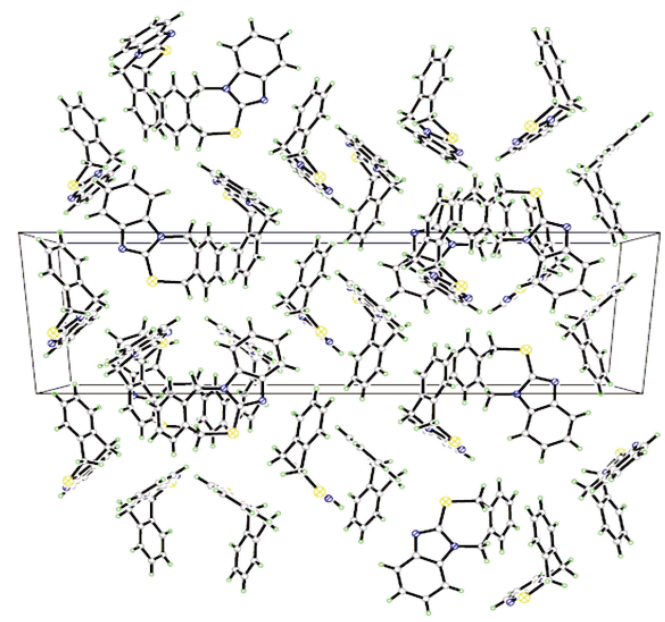

图 2 化合物 1-3a 晶胞堆积图

Figure 2 The accumulation of compound 1-3a

表 1 部分键长 $(\AA)$ 和键角 $\left({ }^{\circ}\right)$

Table 1 Selected bond lengths $(\AA)$ and bond angles $\left({ }^{\circ}\right)$

\begin{tabular}{llllll}
\hline $\mathrm{S}(1)-\mathrm{C}(1)$ & $1.732(6)$ & $\mathrm{N}(1)-\mathrm{C}(7)$ & $1.385(6)$ & $\mathrm{N}(2)-\mathrm{C}(2)$ & $1.388(6)$ \\
$\mathrm{S}(1)-\mathrm{C}(15)$ & $1.824(6)$ & $\mathrm{N}(1)-\mathrm{C}(8)$ & $1.467(6)$ & $\mathrm{C}(8)-\mathrm{C}(9)$ & $1.497(7)$ \\
$\mathrm{N}(1)-\mathrm{C}(1)$ & $1.382(6)$ & $\mathrm{N}(2)-\mathrm{C}(1)$ & $1.326(7)$ & $\mathrm{C}(14)-\mathrm{C}(15)$ & $1.495(8)$ \\
$\mathrm{C}(1)-\mathrm{S}(1)-\mathrm{C}(15)$ & $104.9(3)$ & $\mathrm{N}(1)-\mathrm{C}(1)-\mathrm{S}(1)$ & $128.6(4)$ & $\mathrm{C}(2)-\mathrm{C}(7)-\mathrm{C}(6)$ & $123.0(5)$ \\
$\mathrm{C}(1)-\mathrm{N}(1)-\mathrm{C}(7)$ & $105.9(4)$ & $\mathrm{N}(2)-\mathrm{C}(2)-\mathrm{C}(7)$ & $110.8(5)$ & $\mathrm{C}(9)-\mathrm{C}(14)-\mathrm{C}(15)$ & $119.7(5)$ \\
$\mathrm{C}(1)-\mathrm{N}(1)-\mathrm{C}(8)$ & $128.2(4)$ & $\mathrm{N}(2)-\mathrm{C}(2)-\mathrm{C}(3)$ & $129.7(5)$ & $\mathrm{C}(13)-\mathrm{C}(14)-\mathrm{C}(15)$ & $120.2(5)$ \\
$\mathrm{C}(7)-\mathrm{N}(1)-\mathrm{C}(8)$ & $125.6(4)$ & $\mathrm{N}(1)-\mathrm{C}(7)-\mathrm{C}(2)$ & $105.9(4)$ & $\mathrm{C}(14)-\mathrm{C}(9)-\mathrm{C}(8)$ & $118.9(5)$ \\
$\mathrm{C}(1)-\mathrm{N}(2)-\mathrm{C}(2)$ & $104.2(4)$ & $\mathrm{N}(1)-\mathrm{C}(7)-\mathrm{C}(6)$ & $131.1(5)$ & $\mathrm{C}(14)-\mathrm{C}(9)-\mathrm{C}(10)$ & $119.9(5)$ \\
$\mathrm{N}(2)-\mathrm{C}(1)-\mathrm{N}(1)$ & $113.3(5)$ & $\mathrm{N}(1)-\mathrm{C}(8)-\mathrm{C}(9)$ & $112.6(4)$ & $\mathrm{C}(14)-\mathrm{C}(15)-\mathrm{S}(1)$ & $113.7(4)$ \\
$\mathrm{N}(2)-\mathrm{C}(1)-\mathrm{S}(1)$ & $118.2(4)$ & & & & \\
\hline
\end{tabular}




\section{3 结论}

报道了含硫苯并咪唑环蕃的合成, 产物经 IR、 ${ }^{1} \mathrm{H}$ NMR、HRMS 和 $X$ 射线衍射的证实. 因此将苯并咪唑 及其衍生物引入环蕃结构中, 为环蕃化合物的发展注入 了新的活力, 进一步改善了其仿酶功能. 而含硫的环蕃 类化合物更是因为其不同于一般环蕃的结构特点和电 子特性而表现出一些优越的性质.

\section{References}

[1] Lindoy, L. F. The Chemistry of Macrocyclic Ligand Complexes, Cambridge University Press, London, 1989, pp. $4 \sim 20$.

[2] Lv, G.-H.; Hua, C.-W.; Gou, X.-F.; Liu, Y.-F. Chin. J. Org. Chem. 2010, 30(5), 745 (in Chinese). (吕国华, 花成文, 荷小锋, 刘艳风, 有机化学, 2010, 30(5), 745.)

[3] Yang, B.-Q.; Bai, Y.-J.; Gao, B.; Zhang, B.-L. Acta Chim. Sinica 2010, 67, 1629 (in Chinese). (杨秉勤, 白银娟, 高波, 张秉林, 化学学报, 2010, 67, 1629.)

[4] Xi, H.-T.; Sun, X.-Q.; Meng, Q.; Pan, Y.; Hu, H.-W. Prog. Chem. 2008, 20(1), 87 (in Chinese). (席海涛, 孙小强, 孟启, 潘毅, 胡宏纹, 化学进展, 2008, 20(1), 87.)

[5] Shi, Z. Selected Works on Modern Heterocyclic Chemistry, Shaanxi Science and Technology Press, Xi'an, 1998, pp. 131 189 (in Chinese).

(史真, 现代杂环化学选论, 陕西科学技术出版社, 西安, 1998, 131 189.)

[6] You, J.; Yu, X.; Zhang, G.; Xiang, Q.; Lan, J.; Xie, R. Chem. Commun. 2001, (18), 1817.

[7] Shi, Z.; Wang, J.-H. Chin. J. Org. Chem. 1993, 13(2), 114 (in Chinese).

(史真, 王建华, 有机化学, 1993, 13(2), 114.)

[8] Rajakumar, P.; Raja, S. Tetrahedron Lett. 2009, 50(2), 225.

[9] Rajakumar, P.; Sekar, K.; Shanmugaiah, V.; Mathivanan, N. Bioorg. Med. Chem. Lett. 2008, 18(15), 4417.

[10] Su, X.-Y.; Yuan, Y.; Xie, R.-G. Chem. Res. Appl. 2003, 15(2), 152 (in Chinese).

(苏晓渝, 袁艺, 谢如刚, 化学研究与应用, 2003, 15(2), 152.)

[11] Shi, Z.; Thummel, R. P. J. Org. Chem. 1995, 60(18), 5935.

[12] Baker, M. V.; Bosnich, M. J.; Brown, D. H.; Byrne, L. T.; Hesler, V. J.; Skelton, B. W.; White, A. H.; Williams, C. C. J. Org. Chem. 2004, 69, 7641.
[13] Ivanov, Y. E.; Stepanov, D. E.; Yavolovskii, A. A.; Shishkin, O. V.; D'yakonenko, V. V.; Kamalov, G. L. Russ. J. Gen. Chem. 2012, 82(1), 146.

[14] Hull, R.; Hollywood, F.; Suschitzky, H. J. Chem. Soc., Perkin Trans. 1 1979, (12), 3038.

[15] Jones, R. C. F.; Crumpling, L. J.; Iley, J. N. Arch. Org. Chem. 2011, (4), 84.

[16] Wong, W. W. H.; Vickers, M. S.; Cowley, A. R.; Paul, R. L.; Beer, P. D. Org. Biomol. Chem. 2005, 3(23), 4202.

[17] Nishio, S.; Somete, T.; Sugie, A.; Kobayashi, T.; Yaita, T.; Mori, A. Org. Lett. 2012, 14(10), 2476.

[18] Hausner, S. H.; Striley, C. A. F.; Krause-Bauer, J. A.; Zimmer, H. J. Org. Chem. 2005, 70(15), 5807.

[19] Jeletic, M. S.; Ghiviriga, I.; Abboud, K. A.; Veige, A. S. Dalton Trans. 2010, 39(28), 6393.

[20] Rajakumar, P.; Murali, V. Chem. Commun. (Cambridge, United Kingdom) 2001, (24), 2711.

[21] Hassan, A. A.; Mourad, A. E.; El-Shaieb, K. M.; Abou-Zied, A. H.; Doepp, D. Heteroat. Chem. 2003, 14(6), 536.

[22] Suarez, D.; Laval, G.; Tu, S.; Jiang, D.; Robinson, C. L.; Scott, R.; Golding, B. T. Synthesis 2009, (11), 1808.

[23] Han, Y.; Lee, M. Polym. Prepr. (Am. Chem. Soc., Div. Polym. Chem.) 2007, 48(2), 323.

[24] Lin, K.; Kuo, C.; Kuo, L.; Yang, K.; Leung, M.; Hsieh, K. Eur. Polym. J. 2007, 43(10), 4280.

[25] Liu, S.-Q.; Yang, C.-X.; Li, R.-X. Chem. Eng. (Harbin, China) 2001, 87(6), 17 (in Chinese).

(刘思全, 杨春霞, 李瑞香, 化学工程师, 2001, 87(6), 17.)

[26] Guo, Y.-M.; Li, B.-Z. Acta Chim. Sinica 2007, 65(16), 1567 (in Chinese).

(国永敏, 李宝宗, 化学学报, 2007, 65(16), 1567.)

[27] Liu, F.; Majo, V. J.; Prabhakaran, J.; Castrillion, J.; Mann J. J.; Martinez, D.; Kumar, J. S. D. Bioorg. Med. Chem. Lett. 2012, 22(6), 2173.

[28] Janeczko, M.; Orzeszko, A.; Kazimierczuk, Z.; Szyszka, R.; Baiera, A. Eur. J. Med. Chem. 2012, 47, 346.

[29] Wang, R.; Qian, W.; Bao, W. Tetrahedron Lett. 2012, 53(4), 444.

[30] Alcaide, A.; Llebaria, A. Tetrahedron Lett. 2012, 53(16), 2138.

[31] Bodwell, G. J.; Li, J.; Miller, D. O. Tetrahedron 1999, 55(45), 12939.

[32] Li, M.; Yang, B.-Q.; Yuan, H.-A.; Zhao, W.; Shi, Z. Chin. J. Org. Chem. 2006, 26(2), 189 (in Chinese).

(李敏, 杨秉勤, 袁宏安, 赵炜, 史真, 有机化学, 2006, 26(2), 189.) 